\title{
Bone marrow-derived stem cells preserve cone vision in retinitis pigmentosa
}

\author{
Lois E.H. Smith
}

Department of Ophthalmology, Children's Hospital, Boston, Massachusetts, USA.

\begin{abstract}
Retinitis pigmentosa is a heritable group of blinding diseases resulting from loss of photoreceptors, primarily rods and secondarily cones, that mediate central vision. Loss of retinal vasculature is a presumed metabolic consequence of photoreceptor degeneration. A new study shows that autologous bone marrow-derived lineage-negative hematopoietic stem cells, which incorporate into the degenerating blood vessels in two murine models of retinitis pigmentosa, $r d 1$ and $r d 10$, prevent cone loss (see the related article beginning on page 765). The use of autologous bone marrow might avoid problems with rejection while preserving central cone vision in a wide variety of genetically disparate retinal degenerative diseases.
\end{abstract}

Retinitis pigmentosa is a common label for a heterogeneous group of heritable retinal degenerative diseases that result in progressive visual loss secondary to photoreceptor cell death. Of the 2 photoreceptor cell types in the vertebrate retina (rods and cones), these diseases primarily affect rods; the cones die an "innocent bystander" death. This is reflected in the natural clinical course of retinitis pigmentosa, which usually begins with loss of rod-mediated night vision and advances over the years with progressive loss of the peripheral visual field and, ultimately, the loss of central, cone-mediated vision. There is concomitant attenuation of the retinal vasculature. It is thought that vascular loss follows decreased metabolic demand by the photoreceptors. Currently no definitive treatment for retinitis pigmentosa exists, although nutritional approaches may slow some forms of this disease $(1,2)$.

\section{Potential therapies for retinitis pigmentosa}

Encouraging advances in gene therapy have led to partial reversal of the phenotypic changes observed in some animal models of retinitis pigmentosa, the $r d$ (3) and $r d s$ (4) mouse, the Royal College of Surgeons rat (5) and in the RPE65 pheno-

Nonstandard abbreviations used: Lin ${ }^{-}$HSC, lineagenegative hematopoietic stem cell.

Conflict of interest: The author has declared that no conflict of interest exists.

Citation for this article: J. Clin. Invest. 114:755-757 (2004). doi:10.1172/JCI200422930. type in dogs (6), after viral transfection of photoreceptors or retinal pigment epithelium with the appropriate wild-type gene $(7,8)$. However, specific genetic defects have been found in a relatively small number of retinal degenerative diseases, which thereby limits the potential application of gene therapy for those few patients with a known mutation. The use of trophic factors (9-11) and calcium channel blockers (12) has slowed disease progression in some retinal degeneration models, but in the case of trophic factor treatment, delivery of high-molecular-weight proteins to the retina has presented challenging problems. The use of cells expressing a single transgene encoding a neurotrophic substance (ciliary neurotrophic factor), encapsulated to prevent induction of an immune response, has recently been studied in the clinic, although encapsulated cells still retain the potential for rejection (11). There has been some success with transplantation of neural stem cells, but this approach is limited by political and ethical controversies related to the use of embryonic stem cells, as well as by cell rejection (13).

An attractive therapeutic intervention, short of correcting every individual gene mutation, would be one that affords a generic neuroprotective effect with minimal intravitreal injections and without risk of rejection. Protection of both rods and cones would be optimal, but protection of cones alone would be very beneficial, since the human retina is cone-dominated; these cells, responsible for fine and color vision, are concentrated in the central retina (macula), and if they could be maintained, most patients would have highly functional vision, even in the absence of rods.

\section{Autologous bone marrow-derived stem cells prevent loss of cones}

In this issue of the JCI, Otani and coworkers (14) have explored a novel and widely applicable approach to the treatment of these diseases that may offer cone neuroprotection without immune rejection. This work is based on the prior observations of this group and others showing that adult bone marrow-derived lineage-negative hematopoietic stem cells (Lin ${ }^{-}$HSCs) containing endothelial precursors incorporate into degenerating (or newly forming) vessels and stabilize and rescue retinal blood vessels that would ordinarily completely disappear secondary to photoreceptor cell loss (15-17). In their current study, this group has shown that stabilization of the vasculature with Lin- HSCs, which integrate into and associate with degenerating vessels, can prevent loss of cones in 2 mouse models of retinal degeneration, $r d 1$ and $r d 10$ (14) (Figure 1). This effect was observed following delivery of bone marrow derived from genetically defective mice as well as bone marrow derived from wild-type mice. This observation in mice suggests that in humans, the patient's own bone marrow cells (even from patients with a genetic defect) might provide an efficacious cone neuroprotective effect. This effect might not only preserve central vision but might circumvent many of the unwanted potential side effects associated with the use of viral vectors in long-term gene therapy as well as avoiding the problem of rejection that may result following administration of embryonic stem cells.

\section{Can this mouse study} be applied to patients?

There are many questions and much work that lies between the current study and clinical application; what is the specific 
A

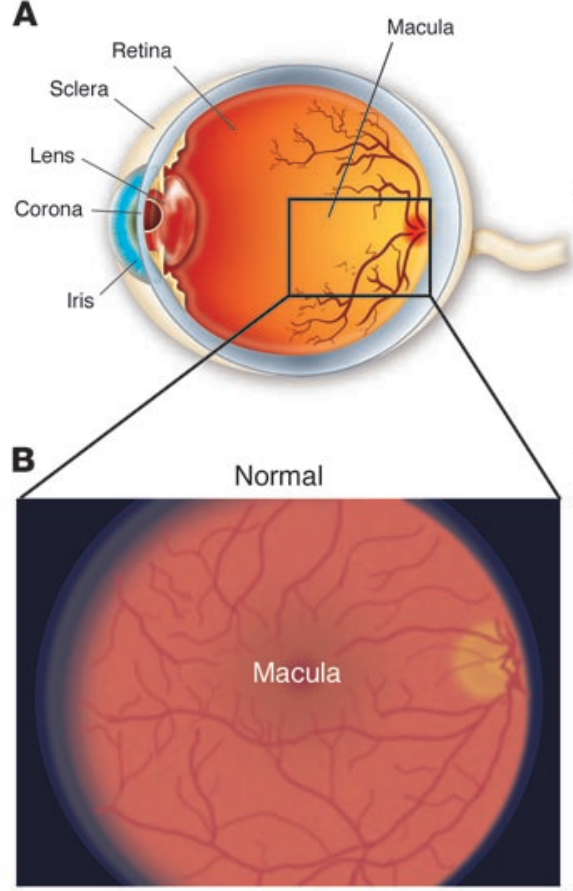

D Adminstration of autologous Lin- HSCs

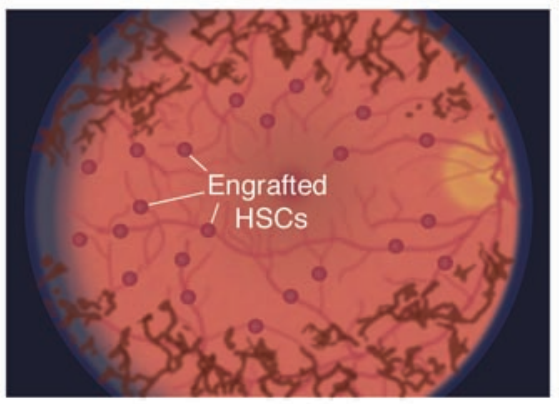

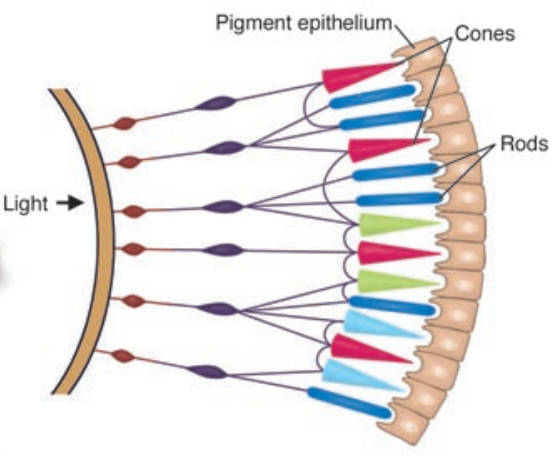

Retinitis pigmentosa

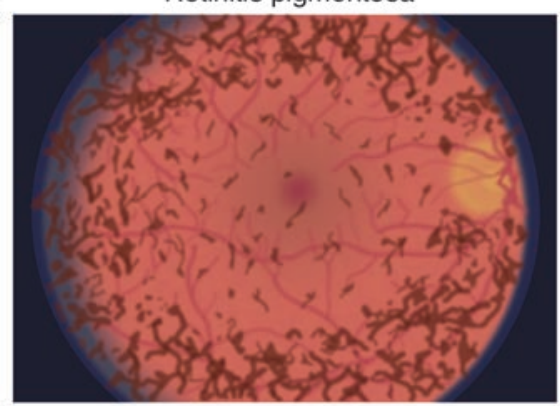

E

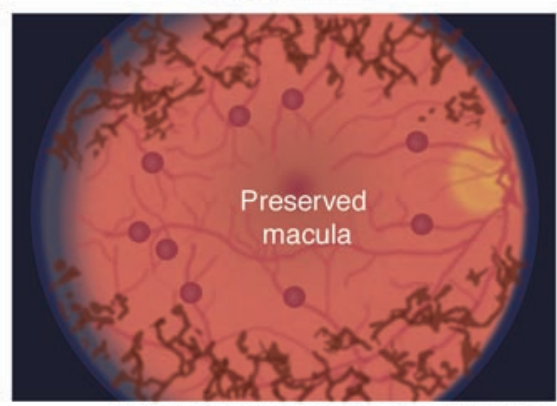

By Lara McCarron and Brooke Grindlinger, J. Clin. Invest.

\section{Figure 1}

Lin- HSCs preserve cones in retinitis pigmentosa. (A) Cross-section of a normal human eye showing vascularized retina with avascular area of central vision (macula). Inset shows enlarged detail of retina with two types of photoreceptors; rods mediate night vision and cones mediate color and central fine vision. (B) View through the pupil of a normal eye showing optic nerve, macula, and vascularized retina. (C) View through the pupil of an eye with late-stage retinitis pigmentosa showing pigmentary changes and attenuated vessels, which occur with photoreceptor loss. (D) Engraftment of Lin- HSCs in blood vessels might prevent loss of cones and preserve macular vision as seen in $\mathbf{E}$. However, there will still be loss of rods, giving rise to pigmentary changes.

cell population in human bone marrow that affords the neuroprotective effect, and can a manufacturing process be developed that would make such a treatment feasible? What is the precise molecular mechanism whereby this effect is achieved, and can it be mimicked by administering trophic substance(s) or small molecule(s)? It is likely that more than one factor is produced by these stem cells to achieve their trophic effect. Are there any long-term toxicities associated with the intravitreal injection of bone marrow-derived Lin $^{-}$ HSCs? How long will such cells produce trophic factors? Will this trophic effect persist after vessels revert to a "normal" mature phenotype, and therefore how often would the injection need to be repeated to achieve long-term stability of the cones? Would autologous bone marrow-derived Lin ${ }^{-}$HSCs engraft in adults as successfully as in the neonates used in these studies, and will these rodent studies be applicable to humans?

We do not know what specifically causes the death of cones in a retina in which a rod-specific genetic defect is present. A rod-produced cone "survival" factor has been identified (18), and if a "cone-friendly" environment could be maintained in the absence of rods, this might be sufficient to maintain functional vision in the human cone-dominated retina. The observation by Otani et al. (14) that a paracrine effect of retinal vasculature could provide such an environment suggests that it may be possible to maintain vision even in the absence of one critical cellular component, such as the rods.

The current study (14) uses a cell-based therapeutic approach based on trophic effects of the natural products of the cells themselves. Using ex vivo transfection with plasmids encoding other trophic substances, it may be possible to achieve an even greater rescue effect (10).

Although the results reported in the study by Otani et al. in this issue (14) suggest a potentially useful approach to preventing cone loss, there are some limitations. This work does not address the issue of rod loss. Nor is the approach useful for diseases that affect cones directly. Those photoreceptors that have already degenerated will not be rescued, and many patients come to the attention of the ophthalmologist late in the disease course. Nor is this paradigm likely to work for patients very early in the course of disease prior to the loss of vessels, since stem cells require a template for engraftment, which is uncovered only after vessels degenerate. Perhaps for those patients, selective ablation of vessels with photodynamic therapy would provide the basis for engraftment of endothelial cell precursors.

Although this study raises many interesting questions, including the question of the precise relationship between retinal neuronal and vascular degeneration, it is not necessary to answer all of them before proceeding further with this new approach to preventing cone loss in retinitis pigmentosa.

Address correspondence to: Lois E.H. Smith, Department of Ophthalmology, Fegan 4, Children's Hospital, 300 Longwood Avenue, Boston, Massachusetts 02115, USA. Phone: (617) 9192529; Fax: (617) 730-0392; E-mail: lois. smith@childrens.harvard.edu. 
1. Berson, E.L., et al. 1993. A randomized trial of vitamin A and vitamin E supplementation for retinitis pigmentosa. Arch. Ophthalmol. 111:761-772.

2. Grant, C.A., and Berson, E.L. 2001. Treatable forms of retinitis pigmentosa associated with systemic neurological disorders. Int. Ophthalmol. Clin. 41:103-110.

3. Takahashi, M., Miyoshi, H., Verma, I.M., and Gage, F.H. 1999. Rescue from photoreceptor degen eration in the rd mouse by human immunodeficiency virus vector-mediated gene transfer. J. Virol. 73:7812-7816

4. Ali, R.R., et al. 2000. Restoration of photoreceptor ultrastructure and function in retinal degeneration slow mice by gene therapy. Nat. Genet. 25:306-310.

5. Vollrath, D., et al. 2001. Correction of the retinal dystrophy phenotype of the RCS rat by viral gene transfer of Mertk. Proc. Natl. Acad. Sci. U. S. A. 98:12584-12589.

6. Acland, G.M., et al. 2001. Gene therapy restores vision in a canine model of childhood blindness. Nat. Genet. 28:92-95.

7. Kajiwara, K., et al. 1991. Mutations in the human retinal degeneration slow gene in autosomal dominant retinitis pigmentosa. Nature. 354:480-483.

8. Travis, G.H., et al. 1991. The human retinal degeneration slow (RDS) gene: chromosome assignment and structure of the mRNA. Genomics. 10:733-739.

9. Frasson, M., et al. 1999. Glial cell line-derived neurotrophic factor induces histologic and functional protection of rod photoreceptors in the $\mathrm{rd}$ / rd mouse. Invest. Ophthalmol. Vis. Sci. 40:2724-2734.

10. Bush, R.A., et al. 2004. Encapsulated cell-based intraocular delivery of ciliary neurotrophic factor in normal rabbit: dose-dependent effects on ERG and retinal histology. Invest. Ophthalmol. Vis. Sci. 45:2420-2430.

11. Uteza, Y., et al. 1999. Intravitreous transplantation of encapsulated fibroblasts secreting the human fibroblast growth factor 2 delays photoreceptor cell degeneration in Royal College of Surgeons rats. Proc. Natl. Acad. Sci. U. S. A. 96:3126-3131.

12. Frasson, M., et al. 1999. Retinitis pigmentosa: rod photoreceptor rescue by a calcium-channel blocker in the rd mouse. Nat. Med. 5:1183-1187.
13. Meyer, J.S., Katz, M.L., Maruniak, J.A., and Kirk, M.D. 2004. Neural differentiation of mouse embryonic stem cells in vitro and after transplantation into eyes of mutant mice with rapid retinal degeneration. Brain Res. 1014:131-144.

14. Otani, A., et al. 2004. Rescue of retinal degeneration by intravitreally injected adult bone marrow-derived lineage-negative hematopoietic stem cells. J. Clin. Invest. 114:765-774. doi:10.1172/ JCI200421686.

15. Grant, M.B., et al. 2002. Adult hematopoietic stem cells provide functional hemangioblast activity during retinal neovascularization. Nat. Med. 8:607-612.

16. Otani, A., et al. 2002. Bone marrow-derived stem cells target retinal astrocytes and can promote or inhibit retinal angiogenesis. Nat. Med. 29:1004-1010.

17. Chacko, D.M., et al. 2003. Transplantation of ocular stem cells: the role of injury in incorporation and differentiation of grafted cells in the retina. Vision Res. 43:937-946.

18. Leveillard, T., et al. 2004. Identification and characterization of rod-derived cone viability factor. Nat. Genet. 36:755-759.

\title{
Prostanoids and blood pressure: which way is up?
}

\author{
Helene Francois and Thomas M. Coffman
}

Division of Nephrology, Department of Medicine, Duke University and Durham Veterans Affairs Medical Center, Durham, North Carolina, USA.

\begin{abstract}
Members of the family of prostanoids, made up of prostaglandins and thromboxanes, are generated via COX-mediated metabolism of arachidonic acid. These lipid mediators exhibit wide-ranging biological actions that include regulating both vasomotor tone and renal sodium excretion. As COX inhibition is often associated with sodium retention leading to edema and hypertension, prostanoids appear to have a role in preventing the development of high blood pressure. On the other hand, prostaglandin $E_{2}$ $\left(\mathrm{PGE}_{2}\right)$ and $\mathrm{PGI}_{2}$ have also been implicated as determinants of renin secretion. A new study suggests that $\mathrm{PGI}_{2}$ plays a critical role in stimulating renin release and promoting hypertension following renal artery stenosis (see the related article beginning on page 805 ).
\end{abstract}

Prostanoids are generated by COX-mediated metabolism of arachidonic acid. These lipid mediators have a myriad of biological actions $(1,2)$. A role for prostanoids in the regulation of blood pressure was originally suggested by early observations that prostaglandins and thromboxanes affect vascular tone and renal excretory functions (3). Later, the impact of prostanoids upon blood pressure in humans was clearly demon-

Nonstandard abbreviations used: EP, E-prostanoid; IP, I-prostanoid;JGA, juxtaglomerular apparatus; NKCC2, Na-K-2Cl cotransporter 2; $\mathrm{PGE}_{2}$, prostaglan$\operatorname{din} \mathrm{E}_{2}$.

Conflict of interest: The authors have declared that no conflict of interest exists.

Citation for this article: J. Clin. Invest. 114:757-759 (2004). doi:10.1172/JCI200422929. strated through clinical experiences with NSAIDs. NSAIDs, among the most widely prescribed drugs worldwide, act by inhibiting COX enzymes and hence blocking prostanoid production. Sodium retention leading to edema and hypertension is often observed in patients treated with NSAIDs $(4,5)$. However, increased blood pressure after treatment with NSAIDs is observed primarily in patients with preexisting hypertension (6-8), suggesting a compensatory role for the prostanoid system in attenuating or preventing the development of high blood pressure.

Data from clinical trials suggest that nonselective NSAIDs, which inhibit both COX isoforms (COX-1 and -2), and selective COX-2 inhibitors (9-12) have similar propensities to cause hyperten- sion. The theory that COX-2-dependent prostanoids resist the development of hypertension is further supported by experiments showing that COX-2 inhibitors or the genetic absence of COX-2 markedly augment the vasoconstrictor actions of Ang II (13). Among the prostanoids, prostaglandin $\mathrm{E}_{2}\left(\mathrm{PGE}_{2}\right)$ and $\mathrm{PGI}_{2}$ are potent vasodilators that promote renal sodium excretion and thus are logical candidates to mediate these counter-regulatory functions. In this regard, studies in humans suggest that COX-2 may be specifically linked to the generation of $\mathrm{PGI}_{2}$ in the systemic circulation (14). The predominant inhibition of $\mathrm{PGI}_{2}$ by COX-2 inhibitors has been posited as an explanation for putative associations between COX-2 inhibitors and the development of cardiovascular complications (15), including hypertension $(16,17)$.

\section{Prostanoids and renovascular hypertension}

In this issue of the JCI, Fujino and associates show that the absence of the receptor for $\mathrm{PGI}_{2}$ (the I-prostanoid [IP] receptor) confers substantial resistance to the development of renovascular hypertension (18). Based on the discussion above, these findings may seem surprising. 